\title{
Prevention of the Paracentesis-Induced Circulatory Dysfunction (PICD) in Cirrhosis: Is the SPA Treatment Worthwhile?
}

\author{
John Carl Hoefs ${ }^{1}$
}

Received: 3 July 2016/ Accepted: 5 July 2016/Published online: 23 August 2016

(C) Springer Science+Business Media New York 2016

The older leading concept concerning ascites formation postulates an imbalance of oncotic and hydrostatic forces, with portal pressure pushing fluid into the peritoneal cavity opposed by the serum colloid osmotic pressure generated by albumin. When hydrostatic exceed oncotic forces, the abdominal cavity fills with fluid until a counterbalancing increase in abdominal pressure prevents further ascites formation. Furthermore, patients with ascites are almost perfectly separated from those without ascites on the basis of portal pressure (relative to the cardiac pressure) and serum albumin concentration [1] seeming to support this concept. According to this model, decreased intra-abdominal pressure due to fluid removal by paracentesis promotes fluid movement into the peritoneal cavity at the expense of the intravascular compartment, potentially resulting in catastrophic hypotension and renal failure. Since these events were thought to occur soon after paracentesis, the prevailing recommendation was for $<2 \mathrm{~L}$ to be removed at any one time. Harmful effects from paracentesis were considered likely despite a large global clinical experience that had not identified a high incidence of hypotension or renal failure due to a common and routine clinical procedure.

Two factors changed this view. The first was the description of oncotic-hydrostatic balance between serum and ascites [2-7] documenting an "abnormal balance" between colloid osmotic and hydrostatic pressure rather than an outright imbalance. In this model, the portal pressure gradient (portal pressure relative to intra-abdominal

John Carl Hoefs

jchoefs@uci.edu

1 Emeritus Professor, University of California, Irvine, Irvine, CA, USA pressure) was the major cause of ascites, as reflected in the serum to ascites albumin gradient (SAAG) both of which are unchanged after removing peritoneal fluid, during diuresis, or following albumin infusions $[4,5]$. Thus, rapid formation of ascitic fluid was an unlikely consequence of paracentesis in this model although a fragile system sensitive to changes in intravascular volume might be predicted. Furthermore, the model predicted the importance of the SAAG in the differential diagnosis of ascites, a widely accepted finding [4, 5] supporting the validity of this model. The second was data obtained from controlled trials that failed to document a major decrease in systemic blood pressure or an increased incidence of renal failure following paracentesis [7]. Indeed, control of ascites with paracentesis appeared safer than with diuretics [7]. Nevertheless, carefully controlled trials using sensitive hemodynamic measures did document statistically significant mild subclinical abnormalities such as alterations in plasma renin activity (PRA) and creatinine in some patients with tense ascites following complete fluid drainage or "total" paracentesis, usually days after the procedure and this is termed paracentesis-induced circulatory dysfunction (PICD) [8, 9]. Progressive renal failure or vascular collapse was not reported in these studies. Nonetheless, the concept of PICD became firmly established, with data supporting the concept that PICD could be prevented by salt-poor albumin (SPA) infusions [9]. The concern remained that some patients with PICD would deteriorate to the point that severe adverse clinical events occurred, justifying the expense of SPA infusions in all patients undergoing paracentesis. Furthermore, similar, but more pronounced alterations were present in the context of this fragile hemodynamic system such as spontaneous bacterial peritonitis, with hemodynamic improvement and increased survival attributed to concurrent SPA infusions [10]. Thus, 
SPA infusions after paracentesis have become the standardof-care.

The majority of the above studies in which PICD occurred came from patients with tense ascites who had undergone "total" paracentesis in which $>8 \mathrm{~L}$ was removed over 20-30 min. In this issue of Digestive Diseases and Sciences, Tan et al. [11] report a study in which the authors slowly removed $<8 \mathrm{~L}$ of fluid at a rate of $65 \mathrm{ml} / \mathrm{min}$ accompanied by SPA followed by a subsequent $<8$-L paracentesis at weekly intervals each followed by additional SPA. They then studied the incidence of PICD based on elevated plasma active renin (PAR), correlating this problem with short- and long-term clinical outcomes. It is somewhat surprising that they had anything to study since PICD should have been prevented completely by the SPA infusions. Of course, the major definition of PICD was based on elevated PAR rather than PRA although the stimuli for PAR and PRA appear to be similar, as discussed by the authors. Furthermore, the serum concentrations of aldosterone and angiotensin II, but not norepinephrine, were elevated 6 days following paracentesis, whereas mean arterial blood pressure (MABP) was slightly decreased, but to the same degree in those with and without PICD, and heart rate was not increased in either group. In follow-up for an average of 2 years, no difference in clinical outcomes was observed between the two groups. The conclusion was that SPA infusions were beneficial in preventing adverse clinical outcomes whether PICD was present or not. The presence of PICD did not identify a group at increased risk of clinical problems in the setting of $<8 \mathrm{~L}$ of fluid removal, slow paracentesis, SPA infusion, and subsequent repeated weekly paracentesis with additional SPA infusions. The lack of a control group did not enable the authors to determine which of the clinical factors accounted for the relatively benign course, failing to provide support for the authors' assumption that SPA infusions were responsible.

The first potential problem with this study was a lack of control group in which SPA was withheld, not included in the study design due to the authors' contention that SPA could not be withheld based on prevailing clinical guidelines [12]. Since the $<8$-L paracentesis and slow removal may have both been beneficial factors, the lack of a control group obscured the conclusion that SPA was beneficial. In actuality, the authors could have withheld SPA from the first paracentesis and then provided SPA with a subsequent paracentesis, serving as an adequate physiologic control for the first paracentesis, using the patient as his own control. Furthermore, universal consensus supporting the administration of SPA after paracentesis is lacking, since adverse clinical events have rarely been reported, unless an elevated PRA is considered a clinical event. Despite this, a meta-analysis of prospective studies of large-volume paracentesis reported a survival benefit for albumin infusions [13]. SPA could benefit a relatively small subgroup with tense ascites rather than benefitting all patients. Perhaps a moderate volume $(<8 \mathrm{~L})$ removed or slow removal alone would decrease the incidence of PICD. A control group is essential for future studies.

The second consideration is the potential confounding effects of diabetes or therapy with $\beta$-receptor antagonists. Although a multivariate post hoc analysis may be of some benefit since these factors were considered unimportant, future trials should adjust for these factors prospectively with $\beta$-receptor antagonists best avoided since they have been associated with PICD [14].

Albumin infusions are still not universally considered to be necessary for all patients. Identification of patients who would benefit the most from albumin and from specific infusion methods would avoid the unnecessary use of this expensive treatment. Since a $<5-\mathrm{L}$ paracentesis appears to avoid the problem of PICD [15], perhaps a $<8-\mathrm{L}$ paracentesis with slow removal is another [11]. In patients identified as requiring repeated large-volume paracentesis, perhaps giving SPA every other paracentesis might be just as effective and save half the SPA.

\section{Compliance with ethical standards}

Conflict of interest I have no conflict of interest.

\section{References}

1. Cerrick GR, Kerr DNA, Read AE, Sherlock S. Colloid osmotic pressure and hydrostatic pressure relationships in the formation of ascites in hepatic cirrhosis. Clin Sci. 1960;19:361-371.

2. Hoefs JC. Serum protein concentration and portal pressure determines the ascitic fluid protein concentration in patients with chronic liver disease. J Lab Clin Med. 1983;102:260-273.

3. Hoefs JC. The mechanism of ascitic fluid protein concentration increase during diuresis in patients with chronic liver disease. Am J Gastroenterol. 1981;76:423-431.

4. Pare P, Talbot J, Hoefs JC. Serum-ascites albumin concentration gradient: a physiologic approach to the differential diagnosis of ascites. Gastroenterology. 1984;85:240-244.

5. Hoefs JC. Globulin correction of albumin gradient: correlation with measured serum to ascites colloid osmotic pressure gradients. Hepatology. 1992;16:396-403.

6. Reynolds TB. Therapeutic paracentesis: have we come full circle? Gastroenterology. 1987;93:386-388.

7. Gines P, Arroyo V, Quintero E, et al. Comparison between paracentesis and diuretics in the treatment of cirrhotics with tense ascites. Results of randomized study. Gastroenterology. 1987;93: 234-241.

8. Ruiz-del-Arbol L, Monescillo A, Jimenéz W, Garcia-Plaza A, Arroyo V, Rodés J. Paracentesis induced circulatory dysfunction: mechanism and effect on hepatic hemodynamics in cirrhosis. Gastroenterology. 1997;113:579-586.

9. Ginès P, Tito L, Arroyo V, et al. Randomized comparative study of therapeutic paracentesis with and without intravenous albumin in cirrhosis. Gastroenterology. 1988;94:1493-1502. 
10. Sort P, Navasa M, Arroyo V, et al. Effect of intravenous albumin on renal impairment and mortality in patients with cirrhosis and spontaneous bacterial peritonitis. N Engl J Med. 1999;341:403409.

11. Tan HK, James PD, Wong F. Albumin may prevent the morbidity of paracentesis-induced circulatory dysfunction in cirrhosis and refractory ascites: a pilot study. Dig Dis Sci. (Epub ahead of print.) doi:10.1007/s10620-016-4140-3.

12. Runyon BA, AASLD Practice Guidelines Committee. Management of adult patients with ascites due to cirrhosis: an update. Hepatology. 2009;49:2087-2107.
13. Bernardi M, Caraceni P, Navickis RJ, Wilkes MM. Albumin infusion in patients undergoing large-volume paracentesis: a meta-analysis of randomized trials. Hepatology. 2012;55:1172.

14. Sersté T, Francoz C, Durand F, et al. Beta-blockers cause paracentesis-induced circulatory dysfunction in patients with cirrhosis and refractory ascites: across-over study. J Hepatol. 2011;55: 794-797.

15. Peltekian KM, Wong F, Liu PP, Logan AG, Sherman M, Blendis LM. Cardiovascular, renal, and neurohumoral responses to single large-volume paracentesis in patients with cirrhosis and diureticresistant ascites. Am J Gastroenterol. 1997;92:394-399. 